\title{
Template-directed carbon nanotube network using self-organized Si nanocrystals
}

\author{
B. Yang, M. S. Marcus, D. G. Keppel, P. P. Zhang, Z. W. Li, B. J. Larson, D. E. Savage, \\ J. M. Simmons, O. M. Castellini, M. A. Eriksson, and M. G. Lagallya) \\ University of Wisconsin-Madison, Madison, Wisconsin 53706
}

(Received 6 May 2005; accepted 17 May 2005; published online 22 June 2005)

\begin{abstract}
We demonstrate a way to direct carbon nanotube growth using Si nanocrystals that are self-ordered via the thermal decomposition of thin silicon-on-insulator substrates. The Si nanocrystals are about $90 \mathrm{~nm}$ wide and 100-150 nm tall, with $200 \mathrm{~nm}$ spacing. Nanotubes connect the silicon nanocrystals to form a network. Nanotubes selectively appear between tops of the Si nanocrystals. We show that the flow pattern of the carbon feedstock in the chemical vapor deposition growth process is disturbed by the geometric effect of the Si nanocrystals, providing a mechanism for growth between the tops of the Si nanocrystals. (c) 2005 American Institute of Physics. [DOI: 10.1063/1.1952585]
\end{abstract}

The bottom-up assembly of nanostructures, including quantum dots, carbon nanotubes, (CNTs), and nanowires, promises a variety of applications ranging from nanoelectronics ${ }^{1,2}$ to nanoelectromechanical systems ${ }^{3,4}$ and sensors. $^{5-7}$ Many studies have focused on directed selfassembly using templates, ${ }^{8-12}$ in which nanostructures are directed to assemble into a particular arrangement when they grow. Such directed self-assembly, including the assembly of carbon nanotubes, has been achieved on nanometer features patterned by advanced lithography (see, e.g., Refs. 9 and 11). Because making such a template involves complicated processing, it is attractive to use self-assembled nanotemplates, if possible.

We describe here the directed self-assembly of nanotubes using such a template, a self-organized nanocrystal array formed via the decomposition of thin silicon-on-insulator (SOI). No lithography is involved. As the Si template layer (the top Si layer) thickness on a SOI (001) wafer is reduced to tens of nanometers, it dewets and agglomerates into $\mathrm{Si}$ nanocrystals at high temperatures in a vacuum. ${ }^{13-15}$ These nanocrystals assemble in a unique fourfold symmetric pattern, ${ }^{13,15}$ with the nanocrystals self-assembled along $\langle 130\rangle$ directions. This arrangement of $\mathrm{Si}$ nanocrystals forms the template for directed assembly of nanotubes. The nanotubes that we grow are primarily suspended between the tops of the Si nanocrystals, even though the catalyst we use is deposited uniformly over the whole surface. We propose a mechanism to explain this observed predominance of CNTs spanning the tops of Si nanocrystals, and support this mechanism with simulations.

A $10 \mathrm{~nm}$ thick Si template layer in SOI dewets and aggregates into self-organized $\mathrm{Si}$ nanocrystals if heated at $950{ }^{\circ} \mathrm{C}$ for $1 \mathrm{~min}$ in an ultrahigh-vacuum environment. A $\mathrm{Fe}-\mathrm{Pt}$ salt solution (following the recipe in Ref. 16, diluted $10^{6}$ times) is spun on or a $1-2 \mathrm{~nm}$ thick Fe film is evaporated on this substrate to act as the catalyst for $\mathrm{CNT}$ growth. Chemical vapor deposition (CVD) is performed at $900{ }^{\circ} \mathrm{C}$, with a flow of $\mathrm{CH}_{4}$ at $400 \mathrm{sccm}$ and $\mathrm{H}_{2}$ at $20 \mathrm{sccm}$ near atmospheric pressure. With this recipe, we have consistently produced CNTs on a variety of surfaces and have examined these with a variety of probes, including transmission electron microscopy, x-ray photoelectron spectroscopy, and elec-

\footnotetext{
${ }^{a)}$ Electronic mail: lagally@engr.wisc.edu
}

trical measurements. Raman spectroscopy measurements show that the nanotube networks contain both single-walled and multiwalled CNTs.

Figure 1 shows a scanning electron microscope (SEM) image of a nanotube network grown using the FePt salt solution. Nanotubes connect the Si nanocrystals and form a network. Usually they extend over several microns, bridging many $\mathrm{Si}$ nanocrystals. Some CNTs, or portions of CNTs, appear fuzzy in Fig. 1(a) because they are not in focus, either because they are higher or lower than the island tops, or because they are vibrating. ${ }^{17}$ Most CNTs are suspended between the tops of $\mathrm{Si}$ nanocrystals. Nanotubes drop to the oxide substrate when the spacing between islands is large, as shown in Fig. 1(b). These observations indicate a strong geometric influence on the nanotube growth. We have achieved similar results on decomposed SOI using a thin Fe film as catalyst (Fig. 2).

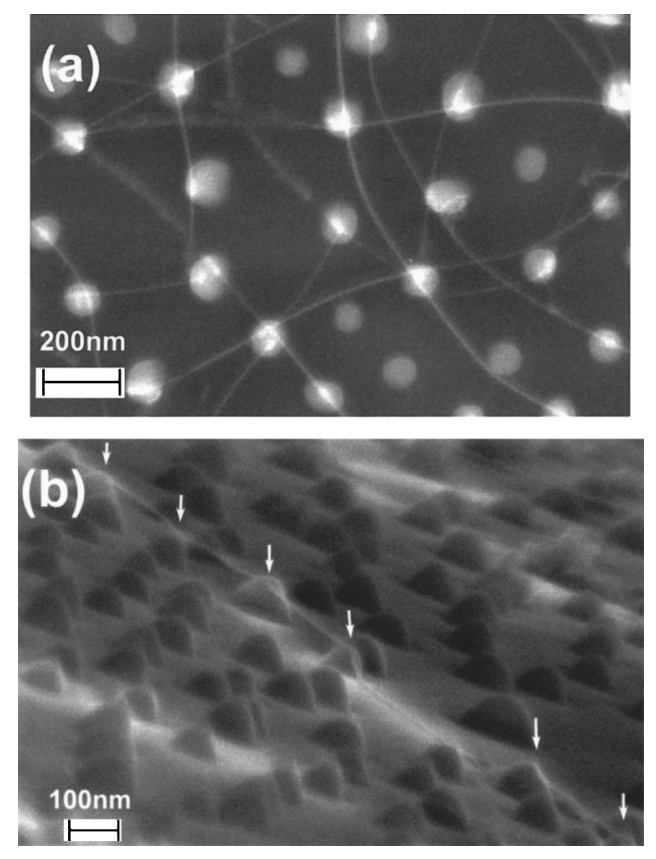

FIG. 1. SEM images of a carbon nanotube network formed on decomposed thin SOI using Fe-Pt solution catalyst: (a) (top view) nanotubes connecting isolated Si nanocrystals and (b) ( $87^{\circ}$ tilted view) suspended nanotube bridging over Si nanocrystals and dipping to the substrate between two widely spaced ones. 

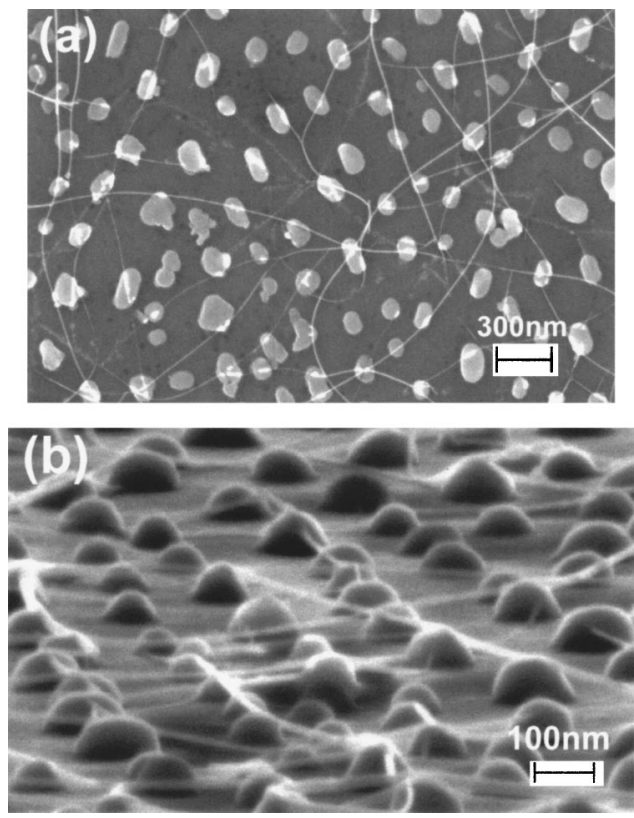

FIG. 2. SEM images of carbon nanotube network on decomposed thin SOI using $1 \mathrm{~nm}$ Fe film catalyst: (a) top view and (b) $80^{\circ}$ tilted view showing a high density of suspended nanotubes.

The nanotube density is sensitive to growth conditions and thus can be adjusted by varying the growth recipe. Under all growth conditions, the majority of the nanotubes is connected to at least one island (Fig. 2). When the nanotube density is low, almost all nanotubes are suspended between the tops of islands. In the tilted view [Fig. 2(b)], as we adjust the focus to shift the focal plane, we can observe that a majority of the nanotubes is suspended between island tops. Among 177 nanotubes counted in SEM images, 4\% sit on the oxide without connecting to $\mathrm{Si}$ islands, while $87 \%$ are attached to at least two $\mathrm{Si}$ islands. The rest are connected to one Si island. The spider-shaped nanotube pattern starting on $\mathrm{Si}$ islands is generally present, suggesting that the growth of the nanotubes starts preferentially from the Si islands. Nanotubes can grow between the islands, reaching neighboring islands, or they can fall to the oxide and stop growing. Of the 121 segments of nanotubes we counted bridging $\mathrm{Si}$ islands whose spacing is smaller than $250 \mathrm{~nm}, 87 \%$ stay suspended between the two islands.

In previous studies using lithographically patterned substrates, where the catalyst is selectively positioned on the top of pillars, nanotubes grow between such pillars. ${ }^{8,18}$ When the catalyst is distributed evenly over the sample, nanotubes grow both on top of the pillars and on the bottom areas between the pillars. ${ }^{11}$ In our experiment, we deposit catalyst over the entire surface and it remains distributed evenly across the substrate, as we observe with SEM at higher magnification, both on top of the $\mathrm{Si}$ nanocrystals and on the oxide between the nanocrystals. Nanotubes can in principle grow anywhere and start from any location, at the top of the islands or otherwise. Nanotubes appear, in fact, to prefer to grow on the oxide. ${ }^{18}$ Yet we observe a strong preference for nanotubes to exist between the tops of the Si islands when the island spacing is small. We suggest that the topography of the nanocrystals on the substrate determines the suspended-nanotube network. We propose that this topography hinders feedstock gas from reaching catalyst particles

reach the bottom areas between the islands. Although there
Downloaded 08 Feb 2007 to 128.104.198.19. Redistribution subject to AlP license or copyright, see http://apl.aip.org/apl/copyright.jsp
FIG. 3. Simulated methane flow pattern on rectangularly shaped island array at $900{ }^{\circ} \mathrm{C}$ : (a) array with $200 \mathrm{~nm}$ spacing, and (b) array with $200 \mathrm{~nm} / 400 \mathrm{~nm}$ spacing. The islands are $90 \mathrm{~nm}$ high and $120 \mathrm{~nm}$ wide. Methane gas flows from the left to the right, with a velocity of $0.0033 \mathrm{~m} / \mathrm{s}$. The top wall is set to be a moving wall in the simulation.

The $\mathrm{Si}$ nanocrystals are about $90 \mathrm{~nm}$ high and 100-150 nm wide, with $200 \mathrm{~nm}$ base-to-base spacing. With such a large height-to-spacing ratio, the nanocrystals may be able to disturb the flow pattern of the carbon feed gas. At our growth conditions, the mean free path of the gas molecules is on the same order as the separation of the Si islands, and the feed gas may not be able to reach the bottom areas between the Si nanocrystals, providing a stagnant zone. To confirm this suggestion, we examine the effect of the Si nanocrystals on the flow pattern for our CVD growth conditions, using computational fluid dynamics (CFD) modeling software (Fluent).

In our CVD growth, the methane flow is laminar. ${ }^{19}$ The furnace tube diameter is much larger than the mean free path of the gas molecules, hence, gas transport is dominated by molecule-molecule collisions, and the flow can be described by continuum theory. As a simple first approximation, we consider only geometric effects in the simulation. The simulation is done in two dimensions $(x, z)$ with an array of rectare $90 \mathrm{~nm}$ high, $120 \mathrm{~nm}$ wide, with $200 \mathrm{~nm}$ [Fig. 3(a)] or $200 \mathrm{~nm}$ and later $400 \mathrm{~nm}$ spacing [Fig. 3(b)], and we simulate ten periods of the rectangular units. The simulation domain height is chosen to be $2 \mu \mathrm{m}$, ten times the island spacing, such that we can still achieve a quantitative picture with reduced computation. The flow front at the entrance of the reactor is uniform in height and a moving boundary with a velocity of $0.0033 \mathrm{~m} / \mathrm{s}$ is placed at the top. Figure 3 shows that the islands markedly disturb the flow pattern; the modified flow is fully developed after the first several islands. Similar to earlier results of flow on rough surfaces, ${ }^{20}$ our simulation reveals stagnant regions (deep blue colored) between the islands and consequently starved nanotube growth conditions [Fig. 3(a)]. The fully developed flow pattern shows that the magnitude of the gas flow velocity between islands is almost zero, so that the methane gas is not able to reach the bottom areas between the islands. Although there angularly shaped islands on the sample surface. The islands 
are catalyst particles on the oxide between the islands, they are not able to capture the carbon feedstock (methane gas) and thus nanotube growth will be starved there. As the island spacing becomes larger, the gas is more able to flow down to the oxide, as shown in Fig. 3(b). Chances for nanotubes to start growing from catalyst particles on the oxide should be larger as the island spacing increases. Indeed, we observe many nanotubes grow from the catalysts on the oxide between the islands when the separation between $\mathrm{Si}$ islands is about $500 \mathrm{~nm}$.

The flow pattern given by the CFD simulation should be qualitatively correct, but it does not take into account the consumption of the feed gas, which will be higher on top of the islands. This consumption of the feed gas further reduces the flow of methane for nanotube growth in the lower regions. We believe this picture explains in a simple physical fashion why nanotubes grow from the tops of the islands when the island spacing is appropriate, without a need to invoke chemical effects. ${ }^{21}$

On a flat substrate, nanotubes generally grow along the substrate surface because of strong van der Waals forces between the nanotubes and the substrate. This strong interaction drives them to maximize their contact area with the substrate. When nanotubes start from catalyst particles on top of the nanocrystals, however, the energy gained by staying close to the substrate comes at the expense of the strain energy for the nanotubes to conform to the substrate morphology. The nanotube profile $h(x)$ along the tube axis is determined by the balance of the strain and adhesion energies $E_{s}$ and $E_{\text {ad }}$. The strain energy $E_{s}$ can be calculated by the integration of $E \pi\left(a^{4}-b^{4}\right) / 8 \int r(x)^{-2} \mathrm{~d} x$ along the tube, where $E$ is the Young's modulus, $a(b)$ is the outer (inner) diameter of the nanotube, and $r(x)$ is the local radius of curvature along the nanotube. ${ }^{22}$ For a nanotube with a diameter of about $100 \AA$, the adhesion energy is reported to be $0.8 \pm 0.3 \mathrm{eV} / \AA{ }^{23}$ The strain energy can be calculated with a known local curvature. It has been shown that a $100 \AA$ nanotube would prefer not to conform to the substrate topography when the island radius is smaller than $500 \mathrm{~nm} .^{24}$ This result qualitatively explains our observations: the nanotubes conform to the three-dimensional (3D) island surface for some length depending on their diameters, and then grow away from the island surface when the strain energy becomes larger than the adhesion energy.

Among the nanotubes that nucleate on tops of the islands, those that manage to stay up and bridge to other islands preferentially continue to grow, while those that dip down fail to survive because their continued growth becomes starved. When the island spacing is small, most of the ones that survive will be bridging. As the spacing gets larger, more nanotubes will start to grow from the catalysts on the oxide surface between the islands because now they are able to capture the feedstock gas. At the same time, nanotubes that nucleate on the islands and grow have a chance to reach down and continue to grow on the oxide. In addition, the nanotubes that are suspended between more widely separated islands have a larger chance to touch the bottom oxide surface and get stuck as they vibrate. Figure 1(b) shows an example: a nanotube is suspended between the tops of several islands, drops onto the oxide surface, and then lies on the tops of the islands again.

In summary, we have produced a carbon nanotube network using a unique nanotemplate, self-assembled Si nano- crystals that form when the thin $\mathrm{Si}$ template layer of SOI decomposes at high temperature under vacuum. No lithography is required. The majority of the nanotubes is suspended between the tops of Si nanocrystals. We explain this behavior by the influence of the island topography on the methane gas flow pattern, with feedstock unable to reach the oxide surface when the 3D islands are close. The nanotubes grow away from the Si nanocrystal surfaces instead of conforming to them in order to minimize the total free energy, and then attach to other nanocrystals. Suspended CNTs grown in this manner may allow us eventually to achieve functional nanotube architectures useful for electronic, optoelectronic, or chemical sensing applications.

The authors thank Prof. S. A. Majetich, Carnegie Mellon University, for providing the Fe-Pt catalysts and Mr. H. F. Tang for help with the CFD simulation. This work was supported by DOE and NSF CAREER program.

${ }^{1}$ Y. Huang, X. F. Duan, Y. Cui, L. J. Lauhon, K. H. Kim, and C. M. Lieber, Science 294, 1313 (2001).

${ }^{2}$ A. Bachtold, P. Hadley, T. Nakanishi, and C. Dekker, Science 294, 1317 (2001).

${ }^{3}$ A. Husain, J. Hone, H. W. C. Postma, X. M. H. Huang, T. Drake, M. Barbic, A. Scherer, and M. L. Roukes, Appl. Phys. Lett. 83, 1240 (2003).

${ }^{4}$ A. M. Fennimore, T. D. Yuzvinsky, W. Q. Han, M. S. Fuhrer, J. Cumings, and A. Zettl, Nature (London) 424, 408 (2003).

${ }^{5}$ O. D. Velev and E. W. Kaler, Langmuir 15, 3693 (1999).

${ }^{6}$ J. Kong, N. R. Franklin, C. W. Zhou, M. G. Chapline, S. Peng, K. J. Cho, and H. J. Dai, Science 287, 622 (2000).

${ }^{7}$ Y. Cui, Q. Q. Wei, H. K. Park, and C. M. Lieber, Science 293, 1289 (2001).

${ }^{8}$ N. R. Franklin and H. J. Dai, Adv. Mater. (Weinheim, Ger.) 12, 890 (2000).

${ }^{9}$ T. Kitajima, B. Liu, and S. R. Leone, Appl. Phys. Lett. 80, 497 (2002).

${ }^{10}$ T. I. Kamins and R. S. Williams, Appl. Phys. Lett. 71, 1201 (1997).

${ }^{11}$ Y. Homma, Y. Kobayashi, T. Ogino, and T. Yamashita, Appl. Phys. Lett. 81, 2261 (2002).

${ }^{12}$ A. M. Cassell, G. M. McCool, H. T. Ng, J. E. Koehne, B. Chen, J. Li, J. Han, and M. Meyyappan, Appl. Phys. Lett. 82, 817 (2003).

${ }^{13}$ R. Nuryadi, Y. Ishikawa, and M. Tabe, Appl. Surf. Sci. 159, 121 (2000).

${ }^{14}$ B. Legrand, V. Agache, J. P. Nys, V. Senez, and D. Stievenard, Appl. Phys. Lett. 76, 3271 (2000).

${ }^{15}$ B. Yang, P. P. Zhang, D. E. Savage, G. H. Lu, M. H. Huang, F. Liu, and M. G. Lagally (unpublished).

${ }^{16}$ Y. Ding, S. Yamamuro, D. Farrell, and S. A. Majetich, J. Appl. Phys. 93, 7411 (2003).

${ }^{17}$ B. Babic, J. Furer, S. Sahoo, Sh. Farhangfar, and C. Schönenberger, Nano Lett. 3, 1577 (2003).

${ }^{18}$ Y. J. Jung, Y. Homma, T. Ogino, Y. Kobayashi, D. Takagi, B. Q. Wei, R. Vajtai, and P. M. Ajayan, J. Phys. Chem. B 107, 6859 (2003).

${ }^{19}$ At atmospheric pressure, the flow velocity $v$ of the methane gas is calculated to be $0.0033 \mathrm{~m} / \mathrm{s}$ (the methane mass flow rate is $400 \mathrm{sccm}$ and the diameter $d$ of the furnace tube is 2 in.). We estimate that the Reynolds number $(\mathrm{Re})$ of methane flow in the furnace tube is smaller than 10 using the formula $\operatorname{Re}=\rho v d / \eta$, where $\rho$ is the density of methane gas ( $\rho$ $=178 \mathrm{~g} / \mathrm{m}^{3}$ at $900{ }^{\circ} \mathrm{C}$ and $\left.1 \mathrm{~atm}\right)$, and $\eta$ is the methane gas viscosity $\left(\eta=1.8 \times 10^{-5} \mathrm{~kg} / \mathrm{ms}\right)$. In addition, the mean free path $\lambda$ of methane molecules is very small compared to the tube diameter at our atmospheric growth condition $\left(\lambda=5 \times 10^{-3} \mathrm{~cm}\right.$ for $\mathrm{N}_{2}$ at room temperature and 1 Torr). The Knudsen number is much smaller than 0.01 . Thus the gas flow can be modeled by continuum theory.

${ }^{20}$ Y. D. Hu, C. Werner, and D. Q. Li, J. Fluids Eng. 125, 871 (2003).

${ }^{21}$ K. Prabhakaran, Y. Watanabe, Y. Homma, T. Ogino, B. Q. Wei, P. M. Ajayan, K. V. P. M. Shafi, A. Ulman, S. Heun, A. Locatelli and S. Cherifi, Langmuir 19, 10629 (2003).

${ }^{22}$ D. J. Barber and R. Loudon, An Introduction to the Properties of Condensed Matter (Cambridge University Press, Cambridge, 1989).

${ }^{23}$ T. Hertel, R. Martel and Ph. Avouris, J. Phys. Chem. B 102, 910 (1998).

${ }^{24} \mathrm{Ph}$. Avouris, T. Hertel, R. Martel, T. Schmidt, H. R. Shea, and R. E. Walkup, Appl. Surf. Sci. 141, 201 (1999). 